Revue européenne des migrations internationales

vol. 32 - $n^{\circ} 3$ et $4 \mid 2016$

30ème anniversaire. Renouveler la question migratoire

\title{
Mattelart Tristan, Médias et migrations dans l'espace euro-méditerranéen
}

Isabelle Rigoni

\section{(2) OpenEdition}

Édition électronique

URL : https://journals.openedition.org/remi/7891

DOI : 10.4000/remi.7891

ISSN : $1777-5418$

Éditeur

Université de Poitiers

\section{Édition imprimée}

Date de publication : 1 décembre 2016

Pagination : 360-365

ISBN : 979-10-90426-29-0

ISSN : 0765-0752

\section{Référence électronique}

Isabelle Rigoni, « Mattelart Tristan, Médias et migrations dans l'espace euro-méditerranéen », Revue européenne des migrations internationales [En ligne], vol. 32 - n³ et 4 | 2016, mis en ligne le 01

décembre 2016, consulté le 14 avril 2022. URL : http://journals.openedition.org/remi/7891 ; DOI : https://doi.org/10.4000/remi.7891 


\section{Notes de lecture}

\author{
Leroy, Delphine (dir.) \\ Leroy, Marie (dir.) \\ Histoires d'écrits, histoires d'exils : pers- \\ pectives croisées sur les Écritures en \\ migration(s). - Tübingen : Narr Verlag, 2014. - \\ $243 \mathrm{p}$. \\ ISBN : 978-3-8233-6917-2
}

Cet ouvrage s'inscrit dans la continuité d'un colloque international qui s'est tenu en 2012 à l'Université Paris 8. Comme le souligne Michel Agier dans sa préface, le sujet n'est pas a priori nouveau et la littérature d'exil a été depuis des décennies à la fois un mode d'expression majeur et un objet de réflexion pour les chercheurs. Ce qui distingue cet ensemble de communications, c'est en premier lieu, une volonté collective qui sert de fil conducteur : construire une analyse à partir de l'exil et non sur l'exil. Cet axiome conduit à lier étroitement cette situation d'exil tant à la théorie qu'à la pratique. Ceci a orienté les directeurs et les contributeurs de l'ouvrage vers une multiplication des angles de vue, des échelles et des temporalités. On pourrait être désorienté par le caractère composite de l'ensemble, mais le projet est sciemment assumé et de plusieurs manières. Tout d'abord en adoptant une définition large de la littérature vue comme l'ensemble des écrits sans exclusion. L'exil est lui aussi défini de façon extensive incluant des marges a priori peu incluses dans sa définition comme l'éloignement des appelés du contingent en Algérie ou l'échange de cartes postales entre patronnes parisiennes et " conchitas " portugaises. Les auteurs sont également des plus divers, des migrants et migrantes, des chercheurs, des artistes. La présentation des contributeurs/trices en atteste, s'y succèdent ethnologues, sociologues, chercheuses en sociolinguistique, historiens, philosophes, traducteurs, plasticiens, photographes, écrivains travaillant dans toute la France ou à l'étranger. Les contributions sont également de tailles variées et de formes diverses. Les questions de la réception (à qui destine-t-on ces écrits et comment sont-ils perçus ?) et aussi de la trajectoire du migrant dans et par l'écriture sont aussi constamment posées. Cette mosaïque mêle ainsi de façon assez rare des textes de chercheurs, des images, des fragments de récits de vie. L'interdisciplinarité est donc de règle et chacun peut $y$ puiser selon ses thèmes de recherche, mais aussi ses curiosités. L'ouvrage est divisé en deux parties intitulées pour la première Écritures en migrations et la seconde Migrations en écritures. Dans la première partie, deux thématiques sont développées sous deux titres : Correspondances et reconfigurations identitaires pour le premier axe et Éclairages pour le second. Une place centrale est ici laissée aux objets par excellence des échanges entre les migrants et entre les migrants et la société d'accueil : la lettre et la langue. Les conditions de I'acte d'écriture, la place de l'ordinaire, de I'intime sont au cœur des échanges épistolaires entre Jacques et Suzelle séparés par la guerre et la distance entre la France et I'Algérie qu'analysent Axel Pohn-Weidinger et Fabien Deshayes. Les héros de Naim Kattan, confrontés à l'autre dans le retour d'exil et le cosmopolitisme de la ville de Montréal s'interrogent sur leurs identités et mettent en acte la posture de l'écrivain migrant " qui ne dispose d'autre arme que celle de la langue ". Celle-ci est aussi omniprésente dans les recompositions de la diaspora juive égyptienne présentées par Michèle Baussant. La partie Éclairages choisit des supports plus surprenants pour I'habitué des ouvrages tirés de colloque : un échange de mails entre Delphine Leroy sociolinguiste et un écrivain puis ceux qui accompagnent la restitution de l'expé- 
rience artistique qui a été menée lors du colloque initial, présentée aussi à partir de quelques-unes des œuvres exposées. Qu'il s'agisse du spectacle vivant ou de l'accrochage d'œuvres, on lit ici et de façon parfois abrupte, les interrogations, les décalages, les cloisonnements entre chercheurs et non-chercheurs. Les constats sont souvent sans appel et soulignent l'incapacité des chercheurs à s'intéresser aux populations migrantes sur lesquelles ils travaillent.

La seconde partie entremêle tout autant témoignages, fictions, représentations. Les temporalités et les aires géographiques sont variées du XVlle siècle au très contemporain, de la Hollande au Sahara occidental en passant par Paris. Une place particulière est accordée à l'écriture féminine souvent négligée. Le volume se conclut sur des expériences d'écriture en analysant toujours les pratiques et les ressources mobilisées par les acteurs. Un ouvrage hétéroclite donc, mais à l'image de la multiplicité des situations et des regards portés sur l'exil depuis l'exil.

Sylvie Aprile

Professeure d'histoire contemporaine IRHiS/Université Lille III

\section{Cunin, Elisabeth \\ Administrer les étrangers au Mexique : migra- tions afrobréziliennes dans le Quintana Roo, 1902-1940. - Paris : Karthala, 2014. - 264 p. ISBN : 978-2-8111-1194-6}

En quelques années, de nouvelles études sur l'immigration centraméricaine ou nord-américaine au Mexique sont venues nuancer l'image d'un pays jusque-là perçu comme une terre d'émigration vers les États-Unis. Malgré la grande diversité d'étrangers accueillis depuis le XXe siècle - au-delà des cas bien connus des réfugiés politiques espagnols et chiliens, des commerçants libanais et hommes d'affaires anglo-américains, des travailleurs asiatiques venus de Corée ou de Chine - I'historiographie demeurait très lacunaire. On ne peut donc que saluer la parution en français de l'ouvrage d'Elizabeth Cunin, intitulé Administrer les étrangers au Mexique : migrations afrobéliziennes dans le Quintana Roo, 1902-1940 qui retrace en six chapitres le processus de peuplement d'un territoire du Sud mexicain. Loin du récit officiel du métissage des populations indigènes et européennes, l'auteur nous plonge dans les archives administratives d'un État-Nation encore fragile et restitue aux migrants noirs originaires du Belize leur place dans le récit local et national.

L'ouvrage s'ouvre sur une excellente introduction qui situe cette étude de la migration bélizienne dans la littérature sur les Amériques noires et les migrations afrocaribéennes. Par-delà son intérêt historiographique, ce chapitre théorique met en perspective, de manière décalée et pertinente, l'idéologie mexicaine issue de la Révolution (1910-1920) en demandant " pourquoi le Mexique a mis en place une des politiques migratoires les plus restrictives d'Amérique latine au début du $X X e$ siècle, alors que le pays connaissait un des taux d'immigration les plus faibles du continent " ? Et de plonger le lecteur dans le récit d'un moment clef de l'histoire mexicaine, moment où se dessine, à la marge du territoire national, la mise en œuvre d'un projet de contrôle politique basé sur le mythe du métissage entre Européens et Indiens. En racontant cette frontière sud, poreuse et porteuse d'un autre métissage, Elizabeth Cunin ancre le processus d'invention du Mexique moderne dans une dynamique plus internationale, tout à la fois interaméricaine et impérialiste.

La gestion administrative de la population afrobélizienne au Mexique est à l'image des asymétries de pouvoir entre le Mexique et les grandes puissances de la fin du XIXe siècle. Conscient de sa fragilité territoriale, le pouvoir central basé à Mexico City œuvre, à partir de 1902, à rattacher le territoire du Quintana Roo au 
Mexique. Mais il fait face à des difficultés quand il faut gérer un territoire qui n'a pas été peuplé. Elizabeth Cunin suit la lente consolidation de l'administration migratoire jusqu'à l'accession au pouvoir du président Lazarao Cardenas qui impose I'État révolutionnaire dans le Sud. En s'appuyant sur de multiples corpus d'archives, le livre décrit l'émergence de l'État du Quintana Roo, des décennies avant Cancún, comme un processus d'exclusion qui culmine dans les années 1940, âge d'or du Parti Révolutionnaire Institutionnel.

Les premiers chapitres décrivent des politiques migratoires décrochées des préoccupations locales de développement et peuplement, à l'origine d'arbitrages de terrain en faveur d'une industrie locale dominée par des Béliziens. Le dépouillement minutieux d'archives révèle la présence discrète, mais continue d'une population noire. Le décalage entre une idéologie de plus en plus hostile à l'immigration noire et les impératifs de développement et peuplement de la frontière sud est bien rendu. Elizabeth Cunin ressuscite un territoire perçu comme sauvage et hostile par les élites de Mexico City, où des hommes d'affaires béliziens organisent pendant des décennies la venue de milliers de travailleurs noirs anglophones. Les autres chapitres suivent l'évolution d'une idéologie souvent contradictoire et d'une législation de plus en plus hostile aux migrants noirs à l'ethnicité souvent dissimulée, ce qui donne lieu à un peuplement noir, mais pas sa pérennisation.

L'un des aspects les plus intéressants de cette étude est qu'il fait ressurgir la population noire du Quintana Roo dans toute sa diversité, sans la cantonner dans un rôle subalterne. Les sources administratives sont enrichies d'autres sources qui permettent de rentrer dans les détails des trajectoires individuelles. Les portraits de J. Martinez Ross (en introduction) ou de I'homme d'affaires Robert Sidney Turton (p. 177 et suivantes) font contrepoint au récit souvent sobre de la migration de masse. Ces portraits donnent à voir les ressources, les compétences professionnelles et l'influence politique et économique de certains migrants béliziens. On devine que pour eux, comme pour de nombreux migrants noirs plus ou moins qualifiés qui tentèrent de régulariser leur présence au Mexique, le Quintana Roo fut une terre d'opportunités.

À l'amnésie de l'histoire officielle du Mexique, Elizabeth Cunin oppose donc une analyse fine des jeux de pouvoir. Notre compréhension du projet national mexicain de métissage en est profondément enrichie. Les politiques migratoires sont saisies dans toute leur complexité : désir d'émulation des États-Unis, porosité des frontières et projection extraterritoriale des Empires, circulations multiples entre Amériques et espace caraïbe et développement économique. Bref, "Administrer les étrangers au Mexique : migrations afrobéliziennes dans le Quintana Roo, 1902-1940" écrit un beau chapitre de l'histoire encore mal connue de l'immigration au Mexique.

Ève Bantman-Masum

Maître de conférences en anthropologie LISST-CIEU/Université Toulouse - Jean Jaurès

\section{Aragón, Argán \\ Migrations clandestines d'Amérique centrale vers les États-Unis. - Paris : Presses de La Sorbonne Nouvelle, 2015. - 265 p. \\ ISBN : 978-2-87854-646-0}

Migrations clandestines d'Amérique centrale vers les États-Unis est un ouvrage qui nous emmène sur la route del Norte qu'empruntent les migrants clandestins centraméricains, point cardinal d'une migration dont l'objectif est d'atteindre les États-Unis. À partir d'une enquête de terrain multisituée - lieux d'origine, de transit, frontaliers et d'arrivée - et d'une trentaine d'entretiens, Argán Aragón réalise une véritable ethnographie de la migration clandestine depuis son point de départ jusqu'à son point d'arrivée. Si 
le voyage de la traversée du Mexique et ses modalités sont au centre de cet ouvrage, ce n'est que pour mieux saisir la rencontre antagonique entre la mobilité, incarnée par les migrants, et la frontière, cet " abcès de fixation " - expression empruntée à Marc Augé - censé bloquer l'arrivée des premiers. Car ce livre n'est pas seulement un livre sur les migrants clandestins, mais aussi une réflexion sur la notion de frontière et de contrôle.

L'ouvrage est divisé en trois parties qui se concentrent chacune sur une étape du voyage : la communauté d'origine, la traversée du Mexique et enfin la frontière des États-Unis.

La première partie intitulée Flux nous emmène au Guatemala, dans le village rural d'El Peñasco, à proximité de la frontière mexicaine. Argán Aragón y décrit l'organisation économique de ce village qui se caractérise par l'agriculture, ou plus précisément la monoculture du café. On comprend que les débuts de la migration à destination des États-Unis sont intimement liés aux variations du prix de cette denrée sur le marché international. La migration joue ce rôle de diversification de la source des revenus pour faire face aux éventuels changements du prix du café. Grâce notamment à la rencontre avec les précurseurs de cette migration, l'auteur nous offre une véritable généalogie de la migration dans ce village. Le cas d'Ireneo Molina (pp. 57-59) est un parfait exemple de ces petites histoires qui influencent la grande histoire d'un lieu. L'auteur poursuit en nous décrivant la création et la consolidation des réseaux de migration de ce village à partir du cas des migrants qu'il a rencontrés aux États-Unis.

La deuxième partie intitulée Traverser une épaisse frontière a pour objet la traversée du Mexique. En premier lieu, Argán Aragón y développe une typologie des migrants selon une variété de critères : genre, capital économique et social, raisons subjectives du départ, etc. À partir de ces données, il identifie le capital mobilité des individus qui conditionnera en grande partie les modalités du voyage à venir : capacité à se procurer ou non un passeur - et son niveau de fiabilité - pour I'ensemble du trajet, à payer les pots-devin, etc. Le quatrième chapitre est construit autour des témoignages que l'auteur a recueillis au cours de son enquête. On y découvre les différents types de parcours et les obstacles à la mobilité auxquels se trouvent confrontés les migrants, mais aussi les stratégies mises en place par ces derniers pour y remédier, quand ils le peuvent.

Enfin, la troisième partie intitulée Des frontières et des hommes se concentre plus sur la frontière étasunienne, sa construction historique et l'évolution de son rapport aux migrations clandestines en provenance du Sud au cours de son histoire. Argán Aragón décrit et analyse les évolutions récentes de cette " frontière spectacle » (p. 159) symbole du contrôle : mur, militarisation et utilisation d'un arsenal technologique de pointe. L'auteur poursuit en analysant le rôle que joue le Mexique dans ce contrôle de la migration clandestine dans une sorte d'externalisation de la frontière nord-américaine. Enfin, il nous propose un inventaire des acteurs qui surgissent de cet enrayement de la mobilité : c'est le cas notamment des groupes de narcotrafiquants qui font régner la terreur sur le chemin et participent à une déshumanisation des migrants élevée à son paroxysme.

Au-delà d'une véritable ethnographie du "voyage clandestin ", ce qui ressort de la thèse d'Argán Aragón, c'est que le contrôle toujours plus sophistiqué des frontières nationales - dont la frontière américaine serait le parangon - et leur externalisation géographique n'ont pas pour résultat de bloquer les migrants clandestins. Elles ne font que déplacer le problème et " enrayer " la mobilité, rendant nécessaire l'invention permanente de nouvelles méthodes, ces dernières étant 
à leur tour conditionnées par les nouveaux acteurs qui se greffent à ces nouvelles réalités. Ainsi, on voit qu'au Mexique, pays de transit, ce sont les organisations criminelles qui ont pris le contrôle d'une grande partie des territoires traversés par les migrants. Les narcotrafiquants sont des acteurs permanents tout au long des témoignages recueillis par l'auteur et c'est sans aucun doute une des transformations les plus marquantes de cette dernière décennie, en même temps qu'une des plus tragiques. Aux victimes du Rio Grande et du désert en territoire américain - ceux qu'on laisse mourir " passivement " (p. 231) -, les victimes, de plus en plus nombreuses, de ces groupes criminels ont ajouté à ce phénomène de la migration clandestine vers les États-Unis les ingrédients de l'horreur et du morbide, dans des actes signifiant la déshumanisation progressive de ces migrants au profit d'un vulgaire statut de marchandises qui, en cas de non-rentabilité, deviennent jetables.

La qualification des migrants clandestins comme " artistes de la frontière " qu'Argán Aragón reprend à Ulrich Beck (p. 225) dans sa conclusion me semble être en ce sens une expression peu adéquate : à la différence de l'artiste, on découvre au contraire au fil des pages que ces migrants ne sont pas les scénaristes de leur migration, ni de la frontière. D'autres acteurs se chargent de ce rôle et l'expérience migratoire de ces migrants, dans sa dimension de voyage, apparaît plutôt comme subie, aux mains des premiers l'auteur insiste d'ailleurs fortement et avec raison sur l'importance des rapports de force tout au long du trajet - et conditionnée par les aléas et les hasards des malheurs rencontrés sur une route où la notion d'arbitraire règne en maîtresse. Alors bien sûr, il y a toujours une marge de manœuvre des migrants pour résoudre par eux-mêmes certains problèmes qui se posent durant leur traversée de " l'Enfer mexicain " pour reprendre l'expression de Rodríguez Oceguera (2012) : le cas des femmes qui font usage de leur corps pour obtenir la protection d'autres voyageurs en est un des exemples les plus marquants. Mais est-ce là « un art » de la migration ? Le parallèle avec le Via Crucis que fait Argán Aragón dans le dernier chapitre du livre me semble au contraire beaucoup plus brillant, beaucoup plus fidèle à la confrontation qu'a eue l'auteur avec son terrain. Ce parallèle a la force de synthétiser la religiosité, omniprésente, des migrants avec leur propre souffrance. Le verbe qui revient le plus pour qualifier cette expérience est celui d'aguantar, difficilement traduisible en français, mais dont les significations les plus justes seraient " résister ", " supporter ", " ne pas laisser tomber ".

L'ouvrage d'Argán Aragón a ce mérite de poser des questions et de prolonger la réflexion et la critique vis-à-vis d'un sujet pourtant maintes fois débattu, mais toujours plus d'actualité. Quelle légitimité ont les frontières nationales dans le monde d'aujourd'hui ? La figure du migrant clandestin sert l'auteur dans sa contraposition de deux réalités incompatibles : la mobilité et la fluidité incarnées par le migrant clandestin d'une part, et l'immobilité et la rigidité incarnées par la frontière d'autre part. Les observations de l'auteur le conduisent à considérer que, dans un monde mouvant, les frontières nationales sont une réalité anachronique dont il faudrait accompagner la mutation progressive par des instances supranationales en charge de la gestion des migrations internationales. On a toutefois l'impression qu'au lieu de reculer, l'idée de nation avance avec l'apparition d'un nombre non négligeable de nouveaux États depuis la fin de l'Union soviétique et des frontières qui vont avec. La résurgence des volontés indépendantistes (Catalogne, Écosse, etc.) et encore plus le risque de voir des nations arabes comme I'Irak, la Syrie ou la Libye collapser vont dans le même sens. Aux souhaits de l'auteur de résoudre ces questions au niveau cosmopolitique, on peut opposer le paradoxe que relevait Geertz (1999) quand il dit que la globalisation de l'économie et des communications 
semble au contraire s'accompagner de la multiplication des conflits et des replis identitaires.

Pour conclure, on a le sentiment d'avoir affaire à un travail de grande qualité, très bien documenté, qui s'appuie sur un grand nombre de lectures et sur un matériau riche. Ce dernier s'incarne dans l'utilisation de témoignages très bien choisis en même temps qu'ils sont un témoignage poignant de ce qui est avant tout une expérience humaine dramatique qui se joue de l'autre côté de l'Atlantique et qui n'est pas sans nous rappeler ce qui se joue aux portes mêmes de l'Europe. Car l'ouvrage d'Argán Aragón a aussi, au-delà de ses qualités scientifiques, une véritable dimension journalistique aidée par une véritable maîtrise de la langue et une qualité d'écriture remarquable. Dans le contexte actuel, où on a l'impression d'assister à une nouvelle ère des migrations marquée du sceau de la violence, la lecture d'un tel ouvrage me semble hautement recommandable.

\section{Références bibliographiques}

Geertz Clifford (1999) Mondo globale, mondi locali. Cultura e politica alla fine del XX secolo, Bologna, II Mulino, $136 \mathrm{p}$.

Rodríguez Oceguera Primitivo (2012) La nouvelle loi sur la migration au Mexique ou " le bon, la brute et le truand ", Hommes \& Migrations, 1296, pp. 64-72.

Michaël Da Cruz

Postdoctorant en sociologie El Colegio de la Frontera Norte, Tijuana

Ortar, Nathalie

La vie en deux : familles françaises et britanniques face à la mobilité géographique professionnelle. - Paris : Éditions Pétra, 2015. $-272 \mathrm{p}$.

ISBN : 978-2-84743-120-9

Si la figure du "travailleur mobile ", figure désormais centrale de la modernité, devient de plus en plus familière, elle a cependant rarement été investie comme objet de connaissance. Définir ces acteurs de l'entre-deux autrement que par l'association entre travail et mobilité expose à de redoutables difficultés, car ils constituent un groupe difficilement saisissable - toutes les classes sociales, une large tranche d'âge et différents types de profession sont concernés. Aussi, c'est tout le mérite de Nathalie Ortar, ethnologue, chargée de recherche du ministère de l'Écologie, du Développement durable et de l'Énergie, que $d^{\prime}$ analyser les supports territoriaux de l'identité personnelle et sociale de ces travailleurs mobiles et d'interroger les conséquences de la mobilité sur la " structure familiale, les relations de genre et l'emploi » (p. 12).

Témoignant d'un souci de cumulativité avec les travaux les plus récents empruntant aux sciences sociales ses principales références, l'auteure déploie sur 272 pages et sept denses chapitres, assortis d'une présentation des enquêtés, les effets de la mobilité géographique sur les scènes familiale et professionnelle du travailleur au regard des évolutions liées à la globalisation qui affectent tant le monde du travail que les modes de vie. À partir d'un solide éclairage théorique finement articulé à des données empiriques inédites, Nathalie Ortar défend l'idée selon laquelle la mobilité professionnelle géographique serait avant tout une affaire de famille et plus précisément une affaire de couple. Le groupe familial élargi est en effet sollicité par le travailleur comme point d'appui dans l'organisation des parcours de mobilités - I'hébergement constituant ainsi une ressource majeure. Les " immobiles " sont aussi fortement solliciteurs de ressources familiales en raison de la logistique qu'implique l'organisation pratique de "la vie en deux". Si la mobilité des uns est ainsi rendue possible par celle des autres, les femmes, à l'exception des cadres, demeurent dans leur grande majorité les « organisatrices du quotidien $»$ (p. 80). 
Mobilisant les ressources d'une ethnographie itinérante menée auprès de vingt-six couples confrontés aux effets du travail à distance, dont onze anglophones et quatre parents divorcés ayant connu le célibat géographique alors qu'ils étaient en couple, l'auteure apporte un renouvellement du regard sur un phénomène qui n'est certes pas nouveau - il existe comme pratique de travail dans de nombreux groupes -, mais dont les formes se sont profondément transformées sous l'effet notamment de la globalisation génératrice de nouvelles normes de travail et de modes de " réalisation de soi ". Distinguer au sein des mobilités spatiales professionnelles, celles qui relèvent d'une " migration de style de vie " (p. 18) apparaît dans cette perspective comme un choix heuristique permettant de contrebalancer les effets liés au travail et dégager les aspects normatifs du phénomène.

À la pluralité des groupes professionnels amenés à se mouvoir fait écho la diversité de leurs mobilités - choisies, nécessitées par la profession ou par les contraintes de délocalisation de l'emploi. Le premier chapitre questionne ainsi l'évidence du lien entre mobilité sociale et spatiale rarement analysé autrement qu'en termes de carrière professionnelle et souligne le poids des critères familiaux et plus largement des modes de vie dans la définition du périmètre de la localisation. Mais comment s'effectue l'entrée en mobilité ? À quelle étape de la carrière et pour quels bénéfices escomptés ? Le deuxième chapitre restitue la complexité des arbitrages qui président à la décision de l'ancrage, met en évidence l'importance de la socialisation à la mobilité souvent transmise comme un capital familial et décrit les tensions entre mobilité et immobilité. Ainsi le travail à distance, sujet auquel est consacré le troisième chapitre, pourrait paraître comme une solution tout indiquée pour assurer un double ancrage sur les scènes professionnelles et familiales. La faible présence féminine interroge toutefois sur les réelles possibi- lités du télétravail à favoriser une meilleure articulation entre ces deux scènes et rend tangible la difficulté à organiser sur le long terme sa vie professionnelle à distance. Le coût des déplacements, les conditions matérielles des circulations, la fréquence et la longueur des périodes d'absence, autant de facteurs de différenciation qui rendent difficile l'appréhension des travailleurs mobiles comme groupe à part entière. Ces derniers se caractérisent cependant par le fait d'activer des compétences et des connaissances, de déployer des routines, d'apprivoiser les transports, bref de partager une condition commune dont rend compte la capacité à Se déplacer, titre et objet du chapitre IV. Si savoir se déplacer implique l'aménagement de l'espace/temps spécifique au déplacement, " habiter entre deux " révèle les différentes stratégies dont usent ces travailleurs pour maintenir dans la durée un mode de vie dual. Afin d'éviter le risque du débordement d'une sphère sur une autre, l'adoption sur la scène professionnelle d'un mode de vie entièrement dédié au labeur permet de contrecarrer les facilités que pourrait offrir une vie à distance et de libérer le temps consacré à la famille. On soulignera également l'aspect novateur des analyses proposées dans ce chapitre sur les modalités d'échanges qui assurent le lien entre les deux univers, familial et professionnel, mais le contraignent aussi du fait des absences qui appauvrissent les échanges. L'examen des conséquences de la mobilité pour " ceux qui restent", objet du chapitre $\mathrm{Vl}$, met en évidence les tensions qui émaillent ces modes de vie. Si les femmes sont particulièrement concernées par les effets de la mobilité sur leurs parcours professionnels, les déplacements constituent néanmoins une solution souvent préférée à la mobilité résidentielle laquelle entraîne une déstructuration de l'ancrage social sans la garantie d'une reconstitution ailleurs. Pour autant, l'absence du conjoint n'a pas seulement des répercussions sur la carrière du partenaire, elle pèse sur l'organisation maté- 
rielle de la famille et a des incidences symboliques quand ce sont les femmes qui partent. Ultime étape et point de départ structurant de ces parcours, le retour chez soi ouvre le dernier temps de l'analyse. La gestion de l'absence passe en effet par ces temps de présence qui maintiennent la stabilité familiale dans la discontinuité et recomposent le quotidien au prix d'une forte disponibilité, condition physique et morale d'une présence renouvelée.

Ainsi se dresse, de chapitre en chapitre, le portrait nuancé et ambivalent de ces sédentaires contraints à se déplacer ; un portrait qui fait d'eux des funambules toujours en équilibre entre deux mondes, deux univers régis par des modalités de présence différentes. Cet équilibre sans cesse (r)établi entre les deux scènes reste néanmoins fragile, car il est exposé aux vibrations liées aux événements imprévus, à la matérialité du quotidien, au poids des absences qui ébranlent ce fil ténu reliant deux sphères d'existence. Au croisement entre une sociologie de la famille, des mobilités et du travail, Nathalie Ortar offre un regard unique et désenchanté sur la pénétration dans les modes de vie des valeurs d'autonomie et de réalisation de soi qui s'expriment par la liberté de choisir son emploi et son lieu de vie au prix parfois de renoncements lesquels diffèrent selon l'appartenance sexuée du travailleur. Si I'on peut toutefois s'interroger sur les modes de sorties - prescrits, consentis ou subis - de la mobilité et des scènes professionnelles ou familiales où ils sont décidés, la force démonstrative du propos conduit le lecteur à un questionnement inédit sur la façon dont l'institution familiale perdure malgré la partition spatiale et temporelle induite par l'éloignement de l'un de ses membres. L'ouvrage, comme l'indique son titre $\mathrm{La}$ vie en deux, est ainsi une invite à repenser l'unité par-delà les multiples distances qui éloignent sans jamais séparer.

Constance De Gourcy

Maître de conférences en sociologie LAMES/Aix-Marseille Université
Laffort, Bruno (Éd.)

Entre ici et là-bas. Des Maghrébins

racontent. - Paris : Karthala, 2014. - 588 p.

ISBN : 978-2-81111-285-1

C'est un véritable défi, autant scientifique qu'éditorial, que Bruno Laffort (maître de conférences en sociologie à l'université de Franche-Comté) relève avec son ouvrage Entre ici et là-bas. Des Maghrébins racontent. Cette collection d'entretiens de Maghrébins de part et d'autre de la Méditerranée, habilement présentés et analysés par l'auteur, dessine un portrait à la fois fascinant et complexe des migrations maghrébines en Europe en donnant toute leur place aux récits des migrants.

Entre 2007 et 2010, Bruno Laffort a pu cordonner le projet de recherche " Nouvelles circulations migratoires en Méditerranée " au cours duquel de nombreux chercheurs et chercheuses (I'auteur de l'ouvrage y compris), de différentes nationalités, ont réuni 102 entretiens avec des migrant-e-s algérien-ne-s, marocain-ne-s et tunisien-ne-s. Les interviews ont été conduites en adoptant la méthode des récits de vie et ont été menées entre l'Espagne, la France, l'Italie, l'Algérie, la Tunisie et le Maroc. L'ouvrage présente une sélection de ces entretiens (trente au total) choisis en fonction de leur capacité à montrer ce qui détermine, accompagne et suit les parcours migratoires.

Dans un souci de rendre compte de différents profils de migrants - jeunes, adultes, hommes, femmes -, ce volume imposant (588 pages) consacre une attention particulière à la question du " départ ", aux raisons qui l'incitent, aux stratégies qui le sous-tendent, aux choix des destinations qui l'inspirent. Le premier chapitre, Nouvelles stratégies de départ, se focalise sur les difficultés des migrants qui " prenant acte d'une fermeture des frontières européennes de plus en plus drastique " (p. 25) se retrouvent à devoir inventer de nouvelles solutions pour arriver en Europe, en mettant parfois 
leur vie en péril. Après avoir retracé rapidement I'histoire récente des politiques européennes d'immigration (pp. 27-30), ce chapitre se concentre sur les départs qui se concrétisent de manière " encadrée " (p. 27) - tel est le cas des jeunes étudiante-s à la recherche d'un futur professionnel à l'étranger - ou bien de manière clandestine - les harragas (terme de l'arabe algérien qui désigne les migrants illégaux). Parmi les thèmes abordés, l'auteur insiste, à juste titre, sur la question des décalages existants entre les milieux sociaux dont les immigrés sont issus et leurs répercussions sur les trajectoires migratoires. Pourtant, cet aspect aurait peut-être mérité une étude plus approfondie en ce qui concerne ses différentes déclinaisons dans les divers pays du Maghreb pris en compte. Dans ce riche chapitre, l'on peut également regretter le fait que le mot harraga ne fasse pas l'objet d'une analyse plus détaillée de son origine et de son étymologie, d'autant plus que le profil de l'immigré clandestin désigné par cette expression est au cœur de l'un des récits les plus passionnants que l'on rencontre dans le livre : celui d'Adel et de son voyage vers I'Italie (p. 67 et suivantes).

Le deuxième chapitre analyse la Diversification des destinations de ces migrants. II est notamment consacré à l'étude des processus qui conduisent les Maghrébin-e-s interviewé-e-s à tourner leur attention non seulement sur la France, mais aussi sur d'autres pays européens comme I'Italie ou l'Espagne, voire non européens comme le Canada. Si l'Espagne et l'Italie sont indiquées comme les alternatives pour les migrant-e-s ayant été refusée-s en France, ce chapitre définit également les raisons pour lesquelles le Canada est devenu l'une des destinations les plus séduisantes. Ce dernier choix migratoire semble être influencé par les modalités, claires et strictes, pour avoir un visa, qui, si obtenu, " a valeur d'installation quasi définitive ", "la citoyenneté canadienne [pouvant] être obtenue au bout de trois ans de résidence " (p. 78). L'on aurait néanmoins aimé en savoir plus sur les raisons qui conduisent les migrant-e-s maghrébin-e-s à choisir la société canadienne, considérée comme un espace d'accueil où les rapports entre populations majoritaire et minoritaire se révèlent selon les interviewés eux-mêmes - moins asymétriques qu'en Europe.

Le troisième chapitre complète l'analyse des " raisons du départ " en abordant le cas des jeunes diplômé-e-s arrivé-e-s (ou ayant l'objectif d'immigrer) en France. L'attention est concentrée sur la "difficulté du provisoire " (p. 192) qui caractérise cette population d'immigré-e-s et les péripéties administratives auxquelles elle se confronte pour l'obtention ou le renouvèlement de son statut juridique en Europe. Ce qui émerge des récits recueillis et de l'analyse de Bruno Laffort, tantôt en filigrane tantôt explicitement, est surtout un désenchantement vis-à-vis du " mythe de l'eldorado français " (p. 262).

Le quatrième chapitre Entre mythe et réalité se penche justement sur l'étude des décalages que l'immigré observe entre ce qu'il (ou elle) imaginait avant son départ et une fois arrivé à l'étranger. À travers les entretiens proposés au lecteur, l'auteur adopte une approche originale de la figure du migrant. Elle est analysée non pas au prisme du " désir de partir " et de rester à l'étranger " coûte que coûte " pour éviter la " honte de l'échec » (p. 291), mais plutôt à travers une lecture des " individualités " des migrant-e-s en Europe et de leurs efforts de s'ouvrir à de nouvelles habitudes de vie. Cette démarche est poursuivie dans le cinquième chapitre Religion, laïcité et bricolages " identitaires " où, néanmoins, certaines notions utilisées par l'auteur restent parfois vagues, comme c'est le cas pour le concept d'individualisation (p. 402).

Le dernier chapitre s'intéresse aux " violences politiques " et à leur influence sur les parcours migratoires des personnes interrogées (p. 465). L'auteur s'attache à décrire la situation actuelle et l'histoire 
récente des pays maghrébins (Algérie, Maroc, Tunisie). II réfléchit sur les questions de la (non) démocratie, des inégalités sociales, de la répression des libertés, ainsi que sur les résistances individuelles et collectives à ces violences politiques. Les entretiens retenus présentent les cas de migrant-e-s algérien-ne-s et tunisien-ne-s, alors que le témoignage d'aucun interviewé marocain n'est présent. Cela révèle la singularité du cas du Maroc qui aurait sans doute mérité une analyse plus précise pour en mettre en évidence les particularités par rapport aux deux autres pays sur lesquels l'attention de l'auteur se concentre davantage.

En se départant d'un regard essentialiste et monolithique sur les phénomènes migratoires en Méditerranée, l'objectif de l'ouvrage de Bruno Laffort est, nous semble-t-il, de combattre les stéréotypes sur les migrant-e-s en les faisant contribuer eux-mêmes à ce combat par leurs récits de vie. L'auteur (p. 23) reprend d'ailleurs les mots de Bertaux (1995) qui, en évoquant La misère du monde de Pierre Bourdieu, écrivait «[...] lorsque le sociologue se met à s'intéresser aux gens, ceux-ci trouvent la sociologie plus intéressante ". Une telle approche permet ainsi à leurs expériences et à leurs récits de devancer, pour une fois, les interprétations du chercheur. $\mathrm{Si}$, au bout du compte, ce choix se révèle être le point fort de ce livre et de son format - c'est exactement la raison pour laquelle nous avons défini le travail de Bruno Laffort comme un défi scientifique et éditorial -, ce même aspect finit parfois par devenir une limite. En effet, le soin avec lequel l'auteur introduit et contextualise les profils des interviewés semble ne pas toujours suffire pour que leurs récits d'entretien offrent un regard exhaustif sur les nombreuses questions, parfois très épineuses, que leurs histoires soulèvent. Par exemple, le cas de Sabina (pp. 39-51) et de son mariage arrangé avec un Français d'origine algérienne - grâce auquel elle pourra quitter son pays et le contrôle envahissant de son père et de ses frères sur ses actions - laisse le lecteur en quête d'une réflexion plus approfondie sur les formes plurielles du pouvoir patriarcal.

Ceci dit, il nous semble important de reconnaître l'audace de Bruno Laffort et sa tentative de revisiter le langage de la sociologie des migrations. Son ouvrage nous éclaire sur les raisons qui influencent les départs des migrants et sur les stratégies adoptées pour trouver une place identitaire loin du pays d'origine. En permettant au lecteur un accès direct à des récits de vie, ce travail révèle les drames qui accompagnent toute migration et qui, trop souvent, sont passés sous silence.

\section{Référence bibliographique}

Bertaux Daniel (1995) Alternatives conceptuelles sur la question du sujet dans la sociologie française, in Roberto Cipriani Dir., Aux sources des sociologies de langue française et italienne, Paris, L'Harmattan, pp. 71-110.

Vulca Fidolini

Docteur en sociologie

Dynamiques Européennes/ Université de Strasbourg

Kante, Seydou

La géopolitique de l'émigration sénégalaise en France et aux États-Unis. - Paris : L'Harmattan, 2014. - $301 \mathrm{p}$.

ISBN : 978-2-343-02967-2

L'émigration sénégalaise fait l'objet ces dernières années d'une attention de plus en plus grande de chercheurs issus de diverses disciplines. Le livre de Seydou Kante, docteur en géographie politique, se propose d'apporter une contribution à la connaissance de cette émigration, à partir d'une étude comparative entre la France et les États-Unis. Trois objectifs sont ainsi visés : rendre compte des caractéristiques et des causes différenciées de l'émigration sénégalaise ; décrire les modalités d'intégration des Sénégalais dans ces deux pays ; étudier les impacts de ces émigrations sur les régions de départ. 
Pour mener à bien sa recherche, Seydou Kante s'est appuyé sur des enquêtes menées en France, principalement en Île-de-France et, dans le cas des États-Unis, à New York et Baltimore. Près de 250 entretiens et une série d'enquêtes par questionnaire, reposant sur des échantillons d'une soixantaine de personnes en moyenne, ont été effectués. Les nombreux tableaux statistiques présents dans le texte sont complétés par quelques trop rares extraits de récits de vie et par une série de photos qui agrémentent la lecture de l'ouvrage.

Partant du constat qu'en 2010 près de 3 millions de Sénégalais vivaient à l'étranger sur une population totale estimée à 12 millions, la première partie de l'ouvrage analyse la dynamique globale de l'émigration sénégalaise dans le monde, afin de contextualiser dans le temps et l'espace les caractéristiques de l'émigration en France et aux États-Unis. Cette géographie des migrations débute par une mise en perspective des nouvelles régions dominantes de départ comme celles de Dakar et du centre-ouest du Sénégal qui ont relayé au second plan les régions de Matam ou Saint-Louis, anciens centre de gravité de la migration internationale (p. 37). L'aperçu de la diversité des destinations géographiques de l'émigration sénégalaise permet, par la suite, de rappeler l'importance du continent africain comme premier lieu de destination, ainsi que l'ancienneté des mobilités vers la France depuis l'époque coloniale. II conduit également à préciser les nouveaux espaces de l'émigration sénégalaise (chapitre $\mathrm{V}$ ) - comme celui des États-Unis à partir des années 1980 lesquels témoignent de la manière dont le champ migratoire est devenu multipolaire.

À cette présentation générale succède la deuxième partie de l'ouvrage consacrée à l'analyse comparative de l'émigration sénégalaise en France et aux États-Unis. Sont d'abord passées en revue les raisons des départs que l'auteur résume, sans véritablement les développer, à quatre motifs principaux : économique, familial, professionnel et artistique. L'analyse des parcours des migrants permet ensuite de montrer que les personnes venues en France et aux États-Unis ont eu le plus souvent une expérience migratoire antérieure en Afrique et en Europe. L'auteur met ici bien en évidence la prépondérance des parcours indirects dans les déplacements, démontrant ainsi toute l'importance d'appréhender la migration dans la durée. Dans cette perspective, Seydou Kante souligne le rôle essentiel des réseaux, en particulier familiaux et amicaux, qui permettent " de trouver des lieux de résidence et de faciliter une insertion professionnelle " (p. 128). L'importance des associations fondées sur différents critères (village, région, national, confrérie ou ethnique) est également mentionnée pour illustrer les modalités d'adaptation et le dynamisme organisationnel des migrants. Celui-ci est particulièrement présent aux États-Unis et trouve à s'exprimer à travers l'extension géographique des Sénégalais dans ce pays (chapitre VIII). Le recensement des principaux sites d'installation (Côte est, Californie, Géorgie, Texas et Floride) met en exergue l'importance de la ville de New York, qualifiée de " capitale de la diaspora sénégalaise en Amérique " (p. 148). On regrette cependant, ici, que ne soient pas analysées les relations entre ces sites ni, curieusement, que ne soit mentionnée la présence de nombreux migrants sénégalais dans I'Ohio. L'approche comparative de cette deuxième partie se conclut sur les caractéristiques sociodémographiques de l'immigration sénégalaise en France et aux États-Unis (chapitre XI). Alors que l'émigration demeure dans les deux pays majoritairement masculine, l'auteur montre bien l'augmentation ces dernières années du nombre de femmes, dont la présence accrue ne doit pas être expliquée uniquement par des raisons familiales, mais également en réponse à des motivations économiques et individuelles. Nombreuses sont ainsi les femmes qui migrent en tant que célibataires pour se 
lancer dans les affaires (salon de coiffure, restauration). L'exemple de ces femmes, ainsi que celui de nombreux migrants qualifiés s'établissant en France ou aux États-Unis, témoignent de nouveaux profils dans l'émigration sénégalaise.

C'est sur les conséquences de cette émigration sur les zones de départ que s'interroge la troisième et dernière partie de l'ouvrage. Seydou Kante rappelle ici l'importance des transferts d'argent destinés essentiellement à la consommation des ménages et à l'investissement immobilier, mais très faiblement aux secteurs productifs. Portant son attention sur les effets négatifs de l'émigration, il en vient à évoquer une double dépendance : celle des émigrés qui ont obligation morale d'envoyer de l'argent et celle de ceux restés au pays qui sont dépendants de ces versements. Cet impact négatif, accentué par la fuite des cerveaux et de la main-d'œuvre qualifiée, conduit l'auteur à conclure sur les mesures prises en vue d'une gestion efficace de la migration sénégalaise, en évoquant notamment les politiques de co-développement conçues autour de trois volets : I'organisation de la migration légale ; la lutte contre l'immigration irrégulière, les actions de développement solidaire.

En résumé, si l'ouvrage a le mérite de donner un aperçu général de l'émigration sénégalaise dans le monde, et plus spécialement en France et aux États-Unis, en revanche, on regrettera les limites des résultats de l'approche comparative, en raison, d'une part, du manque de fiabilité des enquêtes statistiques dont l'auteur reconnaît lui-même " les limites tenant à la taille de l'échantillon et au choix aléatoire des enquêtés " (p. 263) et, d'autre part, de l'absence d'analyse des interrelations entre les sites de migration français et américains. On touche ici sans doute aux difficultés de l'approche multisites qui nécessite du temps et des moyens difficilement gérables à l'échelle d'un seul individu. On regrettera cependant que ces difficultés n'aient pas été quelque peu surmontées par une mobilisation dans le texte de la bibliographie qui omet, par ailleurs, plusieurs références importantes (Stoller, 2002 ; Dia, 2009 ; Kane, 2011). L'ouvrage de Seydou Kante invite donc à poursuivre les investigations sur les transformations en cours dans les migrations sénégalaises dont l'analyse doit contribuer, selon les propres termes de l'auteur, " à comprendre le dynamisme des sociétés et leur devenir » (p. 11).

\section{Références bibliographiques}

Stoller Paul (2002) Money has no smell:The africanisation of New York City, Chicago, Chicago university Press, $232 \mathrm{p}$.

Dia Hamidou (2009) Espaces domestiques, espace villageois, espaces urbains multisitués. Cinquante ans de migrations à partir de la moyenne vallée du fleuve Sénégal (1960-2010), Thèse en sociologie, Université Paris Descartes.

Kane Ousmane (2011) The homeland is the arena: Religion, transnationalism and the integration of Senegalese immigrants in America, Oxford, Oxford University Press, $336 \mathrm{p}$.

Olivier Leservoisier Professeur en anthropologie CEPED/Université Paris Descartes

\section{Schmoll, Camille (dir.)}

Thiollet, Hélène (dir.)

Wihtol de Wenden, Catherine (dir.) Migrations en Méditerranée. Permanences et mutations à l'heure des révolutions et des crises. - Paris : CNRS Éditions, 2015. - 282 p. ISBN : 978-2-271-08558-0

Cet ouvrage propose une réflexion féconde sur les migrations dans l'espace migratoire euro-méditerranéen, champ d'études que la plupart des auteurs ont contribué à définir et à consolider. Les recherches présentées, qui sont en majorité le résultat de longues années de terrain, font apparaître les évolutions et 
les permanences des dynamiques migratoires dans la Méditerranée tout en portant un éclairage sur les faits migratoires contemporains. L'espace méditerranéen est montré parfois comme une ligne de fracture, parfois comme une aire géopolitique avec des frontières problématiques, parfois comme un espace socio-économique multidimensionnel. Sans jamais assumer une position qui essentialiserait son existence, les contributeurs resituent la visibilité actuelle de la Méditerranée sur la scène migratoire mondiale en renouvelant la question de l'importance de la mobilité dans cet " espace mouvement ", comme I'a défini Braudel.

Dès I'introduction, Camille Schmoll, Hélène Thiollet et Catherine Wihtol de Wenden interrogent la spécificité de l'espace migratoire méditerranéen et dessinent trois configurations : I'ensemble Maghreb/Europe, I'ensemble balkanique et l'ensemble proche-oriental. Les études de cas présentés restituent les singularités de ces trois configurations et confirment que, comme le soulignent les coordinatrices, la Méditerranée est un espace d'affrontements et de controverses de voisinage, d'enjeux mondiaux et de conflits. L'histoire de la Méditerranée s'est bâtie sur la circulation d'hommes et de femmes, la mobilité a façonné la sédentarité, les échanges pacifiques et conflictuels ont modelé et remodelé les frontières des territoires de cette région.

Les articles sont regroupés en quatre parties. La partie I : Un contexte institutionnel en mutation ? Structures, institutions, révolutions; la partie II : Borders and boundaries. Espaces, politiques, pratiques du contrôle et de marginalisation ; la partie III : Politiques des flux, politiques des identités : les migrations à l'épreuve des changements politiques et sociaux ; la partie IV : Mobilités polymorphes dans un contexte de changement social. L'ouvrage s'ouvre avec un aperçu statistique de 1950 à 2014 qui confirme que les migrations familiales, qualifiées, irrégulières et saison- nières se sont croisées au cours du temps (Elena Ambrosetti) ; la postface de Nicholas Van Hear rappelle les liens existant entre migrations, révolutions et mobilisations.

Au fil de la lecture, chaque article contribue à composer un cadre explicatif avec ses tendances générales, à disposer la question migratoire au cœur des enjeux politiques sociaux, économiques et identitaires et à interroger les échelles géographiques et historiques agissant dans/sur les faits migratoires.

Les analyses des changements migratoires des pays de vieille émigration comme l'Espagne et l'Italie (Thomas Lacroix et Naïk Miret ; Serge Weber), des complexités migratoires de la Turquie (Stéphane de Tapia ; Shoshana Fine), des pays du Maghreb (Ali Bensaâd) ou encore d'Israël (Lisa Anteby-Yemini et William Berthomière) montrent la coexistence de configurations différentes qui sont traversées par les migrations de retour (Giulia Fabbiano) et les mobilités des étudiants (Antonina Levantino). D'autre part, les continuités avérées entre migrations et révolutions (Delphine Pagès-El Karoui) ne peuvent être étudiées qu'en tenant compte des superpositions existant entre circulations, migrations forcées et différentes formes de migrations familiales et économiques (Kamel Doraï et Cyril Roussel).

Le regard posé sur les pays de la Méditerranée révèle ainsi que les migrations sont multi-polarisées, disposées autour de divers pôles économiques, reliant des pays appartenant à différentes aires géopolitiques, et qu'elles ne peuvent pas être saisies à l'intérieur des limites de chaque État-nation. Les migrations créent de nouvelles centralités politiques - elles s'expriment également dans l'exercice du vote à distance accordé aux migrants (Thibaut Jaulin) - qui interrogent la disposition des rapports de dominations dans cette aire géopolitique. En effet, dans tous les pays de la Méditerranée du Nord, du Sud, du Proche-Orient et des Balkans 
apparaît une ligne de convergence vers la même gestion des migrations qui, toutefois, est marquée par le rôle fondamental de I'Union européenne. Les politiques européennes ont créé un " régime migratoire " déséquilibré, caractérisé par une faible coordination en termes de régulation et de gestion des mouvements et une forte préoccupation du contrôle de ses frontières extérieures (Ferruccio Pastore).

Le trafic de migrants (Anna Triandafyllidou), le traitement des morts (Olivier Clochard et Nicolas Lambert) et du corps des morts (Carolina Kobelinsky) dissimulent difficilement l'inefficacité des contrôles aux frontières et du système de réadmission et, surtout, le fait que l'un et l'autre sont la conséquence d'intérêts stratégiques militaires et économiques multiples (Jean-Pierre Cassarino). De plus, les politiques de contrôles européennes engendrent un effet en cascade : elles déterminent la gestion des migrations dans les réalités locales (Marie Bassi), elles produisent informalisation, criminalisation et exploitation dans des pays dits de transit comme la Libye (Ali Bensaâd), elles engendrent la répression des migrants qui aspirent à partir en Europe depuis les pays du Maghreb (Farida Souiah) et des blocages des migrants en circulation en Afrique méditerranéenne (Mehdi Alioua). Les logiques sécuritaires des migrations ont une correspondance dans les logiques économiques néolibérales. Celles-ci placent l'exploitation de la maind'œuvre étrangère au cœur du marché de travail, notamment des secteurs du care (Elena Ambrosini) et du tourisme (Nathalie Bernardie-Tahir). La gestion des migrations, fonctionnelle à une économie mondialisée segmentée en termes de genre et d'origine, se rapproche ainsi à celle du chômage (Manlio Cinalli).

Les études présentées dans cet ouvrage permettent de constater que dans l'espace méditerranéen les configurations actuelles de circulation, de sédentarisation, de stabilisation et de transit s'enchevêtrent et se superposent aux formes migratoires de circulation, de sédentarisation, de stabilisation et de transit du passé. De ce fait, elles nous invitent à considérer la coexistence, l'imbrication et la superposition de diverses configurations migratoires ; à revisiter les catégories interprétatives utilisées (immigration, émigration, installation, retour, transit, réfugiés, demandeurs d'asile, migrants qualifiés et expatriés, travailleurs établis et travailleurs saisonniers) ; et à dépasser une vision étatique dans l'étude des migrations. En même temps, il faut considérer que les mobilités autour du bassin de la Méditerranée œuvrent entre les niveaux global, national, régional et local, et qu'elles produisent des formes d'hybridation, de créolisation, de métissage et de brassage. Ces processus ouvrent deux questions majeures. Comment les faits migratoires concourentils à la sédimentation de formes d'identification et d'altérisation ainsi qu'à la redéfinition des stratifications sociales, culturelles, économiques et politiques? Dans quelle mesure les études sur les différentes configurations dans la Méditerranée peuventelles contribuer à développer une perspective comparative spatio-temporelle dans les études des migrations ?

Adelina Miranda

Professeure d'anthropologie Migrinter/Université de Poitiers

\section{Pécoud, Antoine}

Depolitising Migration: Global Governance and International Migration Narratives. Basingstoke : Palgrave Macmillan, 2015. $155 \mathrm{p}$.

ISBN : 978-1-137-44594-0

Après une thèse en anthropologie sur la migration turque en Allemagne à Oxford, Antoine Pécoud est recruté à I'UNESCO en tant que spécialiste des migrations afin de contribuer au positionnement de l'agence onusienne dans le champ des migrations. Cette expérience est à l'origine de son livre intitulé Depolitising Migration: 
Global Governance and International Migration Narratives, dont le propos est la déconstruction critique de la production discursive relative aux migrations internationales produite et portée par les institutions internationales. II s'inscrit ainsi dans le prolongement de recherches en sciences sociales analysant de l'intérieur le fonctionnement et la culture d'une institution (OMC, Conseil d'État, HCR).

S'inscrivant dans une démarche ethnologique et linguistique, il dépasse son propre jugement selon lequel ces grands récits " ne constituent pas au premier abord un objet de recherche attractif ", pour dresser un stimulant parallèle avec le rôle des mythes à l'intérieur de toute société. II s'appuie sur un corpus constitué de rapports publiés entre 1994 et 2013 et qui se distinguent par une vision globale des migrations, une dimension internationale, un caractère normatif et qui enfin constituent des produits phares de ces institutions. Ce sont donc des discours produits, validés et assumés par les organisations concernées.

Antoine Pécoud retrace les étapes qui ont conduit à l'émergence de la notion de gouvernance globale des migrations durant le XXe siècle. II met en relief le rôle joué par les organisations internationales après la Seconde Guerre mondiale, puis dans les années 1990 après la chute du mur de Berlin. La production discursive sur la migration s'inscrit alors dans l'internationalisation des migrations lorsque les États sous l'effet de différents facteurs et de la montée de peurs au sein des opinions publiques (contrôle de frontières, fin de la guerre froide, déclin des États et montée du village global, intensification de la dérégulation des marchés, nouveaux modèles de gouvernance) prennent conscience de leur incapacité à réguler unilatéralement les flux migratoires. Ils sont alors conduits à adopter des mesures bi- et multi-latérales. Parallèlement, les organisations internationales en tant qu'« entrepreneur bureaucratique " voient dans l'intégration de la question migratoire à leur champ de compétences ou de son élargissement, une stratégie leur permettant de renforcer leur assise et leur visibilité respective dans un contexte de concurrence interagences. Ainsi une véritable " industrie " des récits sur la migration prend corps et assure sa propre dynamique en la reliant à de nouveaux enjeux et de nouvelles catégories de migrants. Par exemple, le passage du " management des migrations " à la " gouvernance internationale " montre comment la question des droits des migrants est en constante tension avec des enjeux plus fondamentaux aux yeux des États.

Antoine Pécoud explicite le processus de production d'un langage normalisé où les rédacteurs gomment toute aspérité politique et amenuisent la complexité des migrations en anticipant toute formulation susceptible de donner lieu à des critiques, donc à un débat. II en résulte une description simplifiée, mais politiquement acceptable, de la migration. Ces discours constituent un savoir commun de base permettant à l'ensemble des professionnels de la migration d'être intégrés au dialogue alors que les migrations constituent un sujet sensible des relations internationales. D'un point de vue formel, le choix d'un anglais simplifié excluant des références nationales ou culturelles, la possibilité de notions intraduisibles dans une autre langue contribue à transcender les traditions des États et facilite les échanges. Dans la même logique, la question de l'identification de l'auteur du rapport (ou de son absence, ou le fait qu'il n'engage pas la responsabilité de l'agence onusienne) produit une auto légitimation, une auto attribution de sa pertinence et de son universalité. Ce processus de dépersonnalisation contribue d'une part à soustraire ces documents à la controverse puisqu'aucun auteur précis ne peut être attaqué. L'écrit facilite d'autre part la synthèse et le compromis. L'auteur pointe ainsi les ambiguïtés de I'UNESCO qui se définit comme une « agence intellectuelle " en référence à sa tradition d'utiliser 
des travaux de scientifiques, mais devant répondre à des contraintes managériales et bureaucratiques dans un contexte idéologique particulier. Cette tension, qui n'est pas spécifique à cette organisation internationale, soulève nombre de questions : l'usage d'une production scientifique dans un milieu non académique, la vision positiviste des sciences sociales conçues comme un outil de production de solutions à des problèmes de population, le rôle de la recherche en tant qu'instance de légitimation du politique et enfin le passage d'une réalité complexe à une rhétorique simplifiée.

Enfin, Antoine Pécoud questionne l'efficacité réelle de ces récits, cet aggloméra d'idées et de connaissances a-t-il un impact sur le changement politique ou constitue-t-il une vision " romantique " de ce que devrait être le monde ? II propose une grille de lecture en appliquant des concepts anthropologiques, tel le mythe, à ces formes contemporaines de récits, à ce jargon aseptisé, démontrant que la question de fond n'est pas tant leur véracité que la fonction sociale qu'ils remplissent. Bien qu'inspirés par la recherche et plaidant pour un renforcement de la collecte des données, ils ne s'inscrivent pas dans un registre scientifique, ils prétendent énoncer une forme de vérité à laquelle les acteurs doivent adhérer, d'une certaine manière il n'y a d'alternative possible à cette forme de croyance hormis le chaos. Les objectifs des organisations internationales (droits humains, développement, paix) constituent un horizon commun difficilement contestable vers lequel tendent les États, par conséquent leur rôle n'est pas d'être collés à la réalité, mais de proposer une vision universelle ou une histoire possible du monde. Aussi les recommandations vis-à-vis des États peuvent demeurer dans les limbes, sauf si elles entrent en synergie avec les intentions de ceux-ci et leur offrent une forme de légitimation.

Ce discours fédératif cherche à établir la centralité et la normalité des migra- tions internationales dans un monde décrit comme globalisé et multiculturel, à convaincre des retombées positives des migrations pour la totalité des acteurs et de la nécessité d'une gestion internationale. Cette coopération est dès lors présentée comme la seule stratégie viable afin de maximiser les profits de la migration à tous les niveaux et pour tous. La migration est systématiquement présentée comme positive et comme le résultat d'un choix individuel volontaire ce qui contribue à sa dépolitisation. Nécessairement universel ce discours est souple et simplificateur effaçant la complexité des migrations derrière la figure du migrant international. Prenant les atours d'un nouveau langage il doit les convaincre, États, migrants, médias, société civile définis comme des acteurs rationnels, d'être davantage conformes à ces recommandations dans leur propre intérêt. Antoine Pécoud relève les ambiguïtés quant à l'articulation des politiques de développement avec les politiques migratoires, les questions politiques sensibles non résolues dans ces rapports et la tension entre des migrations régulées et l'acceptation d'une vision néolibérale des marchés. Ces non-dits ou ces silences frustrent le lecteur qui lirait avec intérêt une suite à cet ouvrage (trop) synthétique, mais passionnant.

Véronique Petit

Professeure de démographie CEPED/Université Paris Descartes

Mattelart, Tristan (éd.)

Médias et migrations dans l'espace euroméditerranéen. - Paris : Éditions Mare \& Martin, 2014. $-579 p$.

ISBN : 978-2-89934-158-2

Cet ouvrage est le fruit d'un projet de recherche collectif sur "Médias et migrations dans l'espace euro-méditerranéen " (Mediamigraterra) financé par I'ANR de 2009 à 2012. II entend analyser le rôle des médias en amont et en aval de la migration, c'est-à-dire comprendre la 
façon dont ceux-ci impactent la dynamique migratoire avant le départ, autant que la dynamique d'intégration lors de l'installation. Remarquable de par sa densité et la richesse de ses contenus, l'ouvrage de près de 600 pages, composé de seize chapitres, est organisé autour de cinq grands axes qui ont également servi de fil conducteur au programme de recherche : le rôle joué par les médias, dans les pays du Maghreb, dans le désir de partir ; les dispositifs médiatiques mis en place par les États du Maghreb à destination de leurs émigrés ; la mise en place, ou pas, de politiques d'insertion médiatique des populations issues de l'immigration dans les pays d'accueil en Europe du Sud (France, Espagne, Italie) ; les usages des médias et des technologies dans les foyers issus de l'immigration maghrébine en France.

La première partie interroge le lien entre médias et émigration. II se garde toutefois de défendre une vision fonctionnaliste qui postulerait l'idée selon laquelle les médias font l'immigration - en présentant notamment les pays européens comme un Eldorado et en attisant les désirs de consommation - et qui négligerait les facteurs sociaux, économiques et politiques dont on connaît bien le poids. Ainsi, le texte de Ratiba Hadj-Moussa s'intéresse à la façon dont l'usage des télévisions par satellite contribue depuis les années 1990 à une transformation de l'espace public, rendant possible l'expression de critiques à l'égard des gouvernements. D'autre part, elle montre que les images occidentales ne sont plus les seules à séduire au Maghreb, notamment parmi les jeunes générations, et que le discours identitaire sur l'" arabitéislamité " des chaînes panarabes vient contrebalancer les représentations de l'immigration, des musulmans et de I'Islam parfois distordues et simplistes véhiculées par les médias occidentaux. Le texte de Tarik Sabry porte sur les liens existants entre les usages d'Internet, notamment des réseaux sociaux, et les projets migratoires des jeunes Marocains. Dans ses travaux précédents des années 2000, l'auteur avait pris à bras-le-corps la question de la relation entre les programmes télévisuels des grandes chaînes occidentales et le désir des Marocains " d'émigrer vers l'Ouest ". Toutefois, loin de se cantonner à l'émigration physique, il évoquait surtout l'importance de "l'émigration mentale " et " symbolique ". Dans la même veine, il interroge d'une part dans cet article les effets des usages des médias sur les stratégies de mise en œuvre du projet migratoire : récolte et échange d'informations pratiques, mais aussi recherche d'une relation amicale ou amoureuse avec une personne déjà installée en Europe, comme les jeunes générations précédentes le faisaient déjà à travers la presse magazine dans les années 1980 ou les cassettes vidéo dans les années 1990'. D'autre part, Tarik Sabry traite également d'un aspect plus méconnu et moins attendu : celui des usages des réseaux sociaux, en particulier de Facebook, non pas pour communiquer avec l'Occident, mais avec d'autres Marocains à l'intérieur du Maroc. Dans le prolongement de ces réflexions, on pourrait s'interroger sur les possibles effets produits et/ou induits par les relations parfois tendues entre Beurs et Blédards, comme l'a démontré Claire Schiff dans des enquêtes portant sur l'analyse de contenu de forums de discussion en ligne dédiés à la communauté maghrébine en France (Schiff, 2015).

La deuxième partie de l'ouvrage s'inscrit volontairement en porte à faux avec la vision d'Appadurai selon laquelle le monde contemporain serait composé de flux et de circulations, au détriment des organisations stables qui s'en trouveraient de ce fait fragilisées. Les quatre textes qui la composent montrent au contraire la force des États du Maghreb, qui utilisent depuis longtemps les médias comme moyen de gestion des migrations, dans une perspective de maintien des liens

1 À ce sujet, voir le film du réalisateur kurde d'Irak Hiner Saleem (1998) Vive la mariée et la libération du Kurdistan. 
communautaires, à la fois en diffusant leur version du récit national et en incitant les émigrés à réinvestir une partie de leurs revenus dans l'économie nationale. Yvan Gastaut montre, avec l'œil de I'historien, la façon dont l'État algérien s'est servi depuis l'indépendance de tous les moyens disponibles pour communiquer avec ses ressortissants à l'étranger, et ce bien avant la popularisation des télévisions satellitaires et d'Internet, avec un journal comme L'Algérien en Europe. II souligne toutefois les difficultés auxquelles est confronté l'État algérien, dès lors que l'immigration de travail se transforme en immigration de peuplement à partir des années 1980 . Dans le prolongement, Mohamed-Ali Adraoui analyse la manière dont Alger se sert de la télévision par satellite, d'abord grâce à Canal Algérie, pour communiquer avec ses émigrés dans les années 1990, à un moment où, parallèlement, chaînes occidentales, surtout françaises, et chaînes arabophones entrent également en concurrence dans le paysage satellitaire au Maghreb. Dans une offre de plus en plus diversifiée, il s'agit aussi de combattre l'apparition des chaînes berbérophones. Là aussi, d'intéressants parallèles pourraient être soumis à la discussion avec la situation turque et le développement de la chaîne satellitaire TRT-International qui entendait, également dans les années 1990, à la fois maintenir les liens politiques et économiques avec les populations turcophones à l'étranger, mais aussi alimenter l'imaginaire national contre l'émergence médiatique d'identités concurrentielles au premier rang desquelles l'identité kurde, elle-même traversée par différents courants politiques et religieux (Rigoni, 2001 ; de Tapia et al., 1997). Mais c'est sur le Maroc que porte l'article suivant, rédigé par Abdelfettah Benchenna, I'un des États d'émigration ayant certainement mis le plus de moyens institutionnels dans le maintien de ses liens, voire de son contrôle, à l'égard de ses ressortissants et de leurs descendants à l'étranger qui, tous, sont considérés comme des
" sujets du roi ». Comme Canal Algérie, Al Maghribiya, destinée aux émigrés d'Amérique du Nord, est fortement dépendante du pouvoir politique marocain et même longtemps condamnée à rediffuser une sélection de programmes émanant des deux chaînes généralistes $\mathrm{Al}$ Aoula et $2 \mathrm{M}$. Aujourd'hui toutefois, le gouvernement marocain réfléchit à la façon dont il pourrait se servir de cet outil pour augmenter son influence dans le paysage audiovisuel international sans que cela ne lui coûte trop cher en termes de liberté. L'article suivant se focalise pour sa part sur les chaînes panarabes reçues en Europe, la façon dont elles représentent les émigrés d'origine arabe et traitent des questions relatives à l'immigration. Naomi Sakr montre tout d'abord qu'à l'inverse des chaînes satellitaires étroitement contrôlées par les États maghrébins, les chaînes panarabes ne s'intéressent pas prioritairement aux publics d'origine arabe. En revanche, lorsqu'elles s'intéressent aux émigrés arabes, et c'est notamment le cas d'Al Jazeera et d'Al Jazeera Children, ceux-ci sont dépeints tour à tour comme des figures de la réussite - ce qui contrebalance avec l'image véhiculée par les médias occidentaux - et comme des citoyens rendus amers par la situation économique, mais aussi par les contraintes politiques qui leur sont imposées par les gouvernements des pays du Maghreb - ce qui contraste fortement avec le positionnement des médias maghrébins.

La troisième partie de l'ouvrage porte la focale sur la rive nord de la Méditerranée en s'intéressant à la façon dont les médias du sud de l'Europe traitent des questions migratoires. Les auteurs montrent l'impact des différentes politiques d'intégration sur la façon dont les médias parlent de l'immigration et s'adressent aux immigrés. En filigrane, on perçoit, pour chacun des États, les liaisons entre les fondements philosophiques de la nation, les modèles politiques d'intégration auxquels correspondent différents traitements juridiques des populations minoritaires, et le discours 
médiatique ${ }^{2}$. Tristan Mattelart revient d'abord sur la genèse des politiques dites de "diversité " en France, depuis le programme télévisé "Mosaïque ", créé dans les années 1970 à destination des immigrés, jusqu'aux plus récentes mesures en faveur d'une meilleure représentation télévisuelle des minorités (Frachon et Sassoon, 2008 ; Rigoni, 2007). II y est également question de l'« intégration médiatique " des populations de religion musulmane, pour lesquelles l'auteur postule que les politiques publiques à leur égard ne servent pas tant le discours politique sur la " cohésion nationale " que des objectifs pragmatiques en matière de défense des intérêts de la sécurité nationale. Dans le second chapitre, Laura Navarro Garcia met en lumière le cas de l'État fédéral espagnol, dont la temporalité et les modalités des politiques visant à améliorer la représentation des populations issues de l'immigration sont très différentes de celles de la France. L'auteure examine la manière dont cette question a été mise sur l'agenda politique catalan à partir des années 1990, en particulier grâce à des acteurs associatifs et institutionnels supranationaux. Elle souligne par là même le décalage avec les autorités nationales qui accordent peu d'intérêt à cette question, comme l'indique la Loi générale de la communication audiovisuelle de 2010. En Italie, Eugénie Saitta décrit une situation encore plus inquiétante, avec l'inexistence de politiques publiques de représentation médiatique qu'elle explique par deux facteurs principaux, celui de la " commercialisation " accrue du secteur télévisuel qui a contribué à " entraver la mission éducative et de service de la télévision publique italienne ", et celui de I'influence politique de la Ligue du Nord qui a imposé un discours sécuritaire. Pour autant, le paysage n'est pas complètement sombre, les deux auteures modérant leur

2 L'article de Navarro et al. (2011) portait déjà sur cette dimension entre les mêmes trois pays. analyse en relayant quelques initiatives émanant de la société civile ainsi que des journalistes en faveur d'une meilleure image des immigrés et d'une " information non raciste ".

La quatrième partie de l'ouvrage est consacrée à une catégorie de médias longtemps oubliée tant des journalistes que des universitaires : les médias issus de l'immigration. Forts de plusieurs milliers de titres dans les démocraties occidentales ${ }^{3}$, les médias des minorités ethniques représentent pourtant un phénomène important et historiquement ancré, contemporain des grandes vagues migratoires. Dans les années 1990, les chercheurs anglo-saxons et scandinaves sont les premiers à utiliser les termes d'" ethnic media " ou d'" ethnic minority media " pour les qualifier. Plus récemment, la multiplication des médias électroniques a toutefois alimenté de plus en plus de recherches, notamment au sud et à l'est de l'Europe, sur ces productions médiatiques ethniques, dont la $R E M I$ et e-migrinter se sont notamment fait l'écho (Cossée et al., 2013 ; Rigoni et al., 2010 ; Rigoni, 2010). Dans cette partie, le texte introductif d'Eugénie Saitta rappelle à juste titre que le propos n'est pas d'« uniformiser " l'ensemble constitué par ces médias ni de " durcir artificiellement l'opposition » entre eux et les médias dominants, comme le soulignait déjà l'ouvrage que nous avions coordonné en 2012 (Rigoni et Saitta, 2012). L'auteure poursuit son analyse en prenant le cas de deux mensuels produits en Italie: Yalla Italia, issu du milieu associatif et consacré aux jeunes d'origine arabe et/ou musulmane, mais s'adressant en italien à un public plus large, et Al Maghrebiya, consacré à la même population, mais rédigé uniquement en arabe et dépendant

3 L'équipe Minority Media a recensé en 2007 plus de 5000 médias dans huit pays européens (programme européen, FP6, 2006-10). Aux États-Unis, The American Newspaper Publishers Association estimait en 1990 que les minorités ethniques composaient $18 \%$ de l'industrie de presse et $16 \%$ de la force de travail. 
d'un groupe de presse commercial. Utilisant les outils de la sociologie du journalisme, elle s'interroge à la fois sur leurs discours et sur les modalités d'accès à ces médias des personnes qui s'y expriment. Dans la même veine, en s'appuyant sur les " médias nés des migrations marocaines en Espagne ", Laura Navarro Garcia aborde les tensions entre le rôle alternatif que disent tenir nombre de ces médias et les logiques économiques et politiques qui contraignent leur action. Surtout, grâce à la perspective socio-historique présente tout au long de son article, l'auteure nous permet de comprendre le processus de création de ces médias dès les années 1970 dans ce jeune pays d'immigration, ainsi que ses développements ultérieurs liés aux influences de plusieurs types de militantisme et aux tentatives d'influence du royaume chérifien. Les deux chapitres suivants, rédigés par Karima Aoudia et consacrés à Radio Beur/Beur FM et à Radio Orient, se font également l'écho à ces réseaux d'influence des États dont sont originaires les populations immigrées, bien décrites par ailleurs dans la sociologie de l'immigration consacrée aux réseaux associatifs immigrés. En dépit des différences de ces deux radios sur leurs origines et leur fonctionnement, l'auteure met également en exergue des points communs parmi lesquels leurs tentatives de gagner un auditoire au-delà de I'Hexagone, élargi aux populations du Maghreb. Le dernier texte de cette partie traite lui aussi des velléités transnationalistes de certains médias minoritaires. En s'appuyant sur l'exemple du portail Yabiladi. com, Abdelfettah Benchenna analyse la force de ce type de dispositif réticulaire en matière de (re)construction de réseaux transnationaux et de stratégies d'influence de part et d'autre des deux rives de la Méditerranée.

La dernière partie de l'ouvrage accorde la part belle aux audiences en s'intéressant aux expériences médiatiques et communicationnelles des publics issus de l'immigration. La question de la réception télévisée au sein des foyers de l'immigration, depuis le développement des antennes paraboliques jusqu'à celui des chaînes d'information arabophones, a suscité au cours de ces dernières décennies en France, des craintes nourries par des questions liées tant aux événements internationaux qu'à la politique intérieure. Plus récemment, la question de l'accès potentiel à des sites islamistes radicaux a contribué à alimenter le débat tout en le déplaçant vers un traitement politique global toujours plus sécuritaire. Les crispations étant moins affirmées en Espagne et en Italie, c'est sur la France que se focalisent les deux textes de ce chapitre, écrits parTristan Mattelart, Elyamine Settoul et Karima Aouidia. Dans la continuité de travaux antérieurs, ils soulignent la grande hétérogénéité des pratiques communicationnelles et informationnelles au sein des foyers issus de l'immigration. Le facteur générationnel est avancé comme l'un de ceux permettant le mieux d'expliquer la différenciation de ces pratiques. À ce titre, le premier chapitre décrit l'élaboration de complexes négociations, au sein du foyer, pour l'accès aux programmes télévisuels préférés et comment les ordinateurs constituent une ressource pour les jeunes. Les auteurs s'attachent également à déconstruire les analyses avancées par d'autres chercheurs sur l'impact des chaînes satellitaires, notamment panarabes, dans I'« économie des pratiques communicationnelles ", en soulignant le rôle joué par les médias du pays de résidence en matière de consommation médiatique. Enfin, le dernier chapitre déconstruit quant à lui le caractère novateur d'Internet, en rappelant les multiples pratiques de mise en contact à distance des familles séparées, que d'aucuns nomment aujourd'hui transnationales. Si là aussi, un pan de la sociologie des migrations s'y était déjà attaché depuis la fin des années 1990, ce texte a pour intérêt de confirmer l'existence, depuis des décennies, d'une économie de la mise en relation transnationale qui s'est successivement appuyée sur différents supports médiatiques. 
Les textes réunis dans cet ouvrage dirigé parTristan Mattelart, articulés autour des flux migratoires et médiatiques traversant un cadre spatial centré sur le pourtour méditerranéen comme le résume Alec Hargreaves dans sa postface, aident à comprendre l'actualité des migrations et des communications médiatiques tout en ancrant les questions qui leur sont liées dans une perspective historique contemporaine. Migrations et médias connaissent des mutations importantes depuis ces trois dernières décennies, renouvelant les conditions dans lesquelles s'élaborent les projets et se déroulent les expériences migratoires dans les pays de départ ainsi que les processus d'inclusion sociale dans les pays d'accueil. De multiples facteurs sont traités, allant des mutations politiques et sociales sur les deux rives de la Méditerranée, aux mutations technologiques qui ont pu confirmer certains processus de production et de consommation médiatiques en cours. Toutefois, si les flux migratoires tout autant que les flux communicationnels s'inscrivent dans une perspective de facto transnationale, cet ouvrage nous invite plus que jamais à prendre la mesure de l'importance des contextes locaux et des interactions localisées.

\section{Références bibliographiques}

Cossée Claire, Navarro Laura, Rigoni Isabelle et Saitta Eugénie (Éds.) (2013) Genre et imbrication des rapports de domination dans les médias des minorités ethniques, e-migrinter, 10, $100 \mathrm{p}$.

Frachon Claire et Sassoon Virginie (Éds.) (2008) Médias et diversité. De la visibilité aux contenus. État des lieux en France, au Royaume-Uni, en Allemagne et aux ÉtatsUnis, Paris, Karthala, 168 p.

Navarro Laura, Rigoni Isabelle et Saitta Eugénie (2011) Exprimer la diversité. Les médias des minorités culturelles et linguistiques en Espagne, en France et en Italie, in Annie Lenoble-Bart et Michel Mathien Éds., Les médias de la diversité culturelle dans les pays latins d'Europe, Bruxelles, Bruylant, pp. 55-69.
Rigoni Isabelle (Éd.) (2010) Migrants, minorités ethniques et Internet. Usages et représentations, Migrations Société, 22 (132), $196 \mathrm{p}$.

Rigoni Isabelle (Éd.) (2007) Qui a peur de la télévision en couleurs ? La diversité culturelle dans les médias, Montreuil, Aux Lieux d'Être, $332 \mathrm{p}$.

Rigoni Isabelle (2001) Mobilisations et enjeux des migrations turques en Europe de l'Ouest, Paris, L'Harmattan, $463 \mathrm{p}$.

Rigoni Isabelle, BerthomièreWilliam et Hily Marie-Antoinette (Éds.) (2010) Les médias des minorités ethniques. Représenter l'identité collective sur la scène publique, Revue Européenne des Migrations Internationales, 26 (1), pp. 7-139.

Rigoni Isabelle and Saitta Eugénie (Eds.) (2012) Mediating cultural diversity in a globalised public space, Basingstoke, Palgrave Macmillan, $187 \mathrm{p}$.

Schiff Claire (2015) Beurs et Blédars, Bordeaux, Le Bord de l'Eau, 260 p.

Tapia Stéphane de, Charef Mohammed et Gauthier Catherine (1997) La circulation migratoire dans les diasporas ouvrières de la Méditerranée, Poitiers, Rapport Migrinter pour le ministère de l'Éducation nationale et de la Culture [non publié].

\section{Isabelle Rigoni \\ Chercheure, Centre Émile Durkheim \\ (UMR-CNRS, Bordeaux) \\ Maître de conférences en sociologie, INS HEA Suresnes}

\section{Simon, Gildas (dir.) \\ Dictionnaire des migrations interna- tionales. Approche géohistorique.- Paris : Armand Colin, 2015. - 807 p. \\ ISBN : 978-2-200-25012-6}

Le géographe Gildas Simon, fondateur du laboratoire Migrinter (Migrations internationales, espaces et sociétés) et de la REMI (Revue Européenne des Migrations Internationales), connu comme un des meilleurs spécialistes des déplacements de populations à l'échelle planétaire, a dirigé 
la rédaction d'un monumental Dictionnaire des migrations internationales qui fait date. Cet ouvrage couvre 190 États ou, dans le cas de la France, départements d'outremer. Les textes, toujours substantiels, ont été rédigés par 150 auteurs, historiens, géographes, démographes, sociologues, anthropologues, politologues. Un système de renvois permet de circuler entre les divers pays liés par le phénomène migratoire.

Le dictionnaire privilégie pour chaque pays une approche géohistorique, située dans le temps long, des grandes découvertes à nos jours, et juxtapose systématiquement deux développements, l'un consacré à l'immigration, l'autre à l'émigration. Toutes les formes de mobilité sont prises en considération, volontaire ou forcée, pacifique ou violente, traditionnelle ou récente, liée à la recherche d'un travail ou d'un asile politique. Aussi trouvera-t-on des mises au point sur la saignée démographique infligée à I'Afrique depuis le XVle siècle par la traite esclavagiste, sur les déplacements de personnes entraînés par les partages de la Pologne et la dictature des Khmers rouges, sur les migrations de la pauvreté, le reflux des pieds noirs vers la France métropolitaine en 1962 et des Européens du Zimbabwe après 1970, l'exil forcé de la moitié des habitants du Liberia durant la guerre civile de 1989-2003, les exodes dus aux affrontements interreligieux en Centrafrique, etc. La dimension culturelle est présente avec les analyses consacrées aux étudiants et surtout à la formation de vastes aires linguistiques, anglophone, francophone, hispanophone, lusophone, en lien avec les migrations anciennes des Européens. Les auteurs mobilisent toutes les ressources documentaires disponibles pour donner à leur raisonnement un fondement scientifique solide. Cependant, ils ne trouvent pas toujours des informations fiables et admettent ponctuellement l'imprécision des connaissances. Si certains pays possèdent des statistiques sûres et anciennes - la Suède est un modèle en la matière -, d'autres ne proposent que des approximations. Ainsi, le nombre des émigrés originaires de la République démocratique du Congo serait compris entre 500000 et 6 millions; la diaspora bangladaise comprendrait 7 à 15 millions de personnes; les Indonésiens de l'étranger seraient entre 4,5 et 6,5 millions d'individus.

L'ouvrage permet de comparer les divers pays et de faire apparaître des tendances générales. Certains États perpétuent au fil du temps une forte tradition d'émigration. Tel est le cas du Portugal : en regard de sa population qui s'élève à 10,4 millions en 2013 , quelque 5 millions de ressortissants, dont 1,9 million nés dans la mère patrie, vivent à l'étranger ; de 1960 à 1973, plus de 1,2 million de personnes, voulant échapper à la dictature, à l'engagement dans les guerres coloniales et à la misère, quittent le pays, surtout en direction de la France ; les départs reprennent de l'importance avec la crise financière de 2008 et atteignent le chiffre de 100000 en 2012. L'émigration récente concerne particulièrement les pays de l'ancien bloc soviétique. En Lituanie la population baisse de 500000 habitants en 1989 et 2011. En Lettonie, le phénomène d'émigration prend une telle ampleur qu'il compromet la reproduction de la population, le développement économique et la pérennité du système de protection sociale. Entre 1991 et 2013 , la Bosnie-Herzégovine perd plus du quart de ses habitants, soit 1 million de personnes.

Le regard vers le passé montre que les départs, notamment depuis l'Europe, ont pris une telle ampleur qu'ils ont littéralement donné naissance à certains pays. Bien connu est le cas du Canada qui continue à favoriser les entrées au rythme de 200000 à 250000 par an, au point qu'aujourd'hui $20 \%$ de la population canadienne est née à l'étranger. En Nouvelle-Zélande la proportion atteint $25 \%$. Les États-Unis, même s'ils sont moins ouverts actuellement, sont nés sur le même modèle et ont 
reçu 33 millions de migrants européens entre 1821 et 1924. Cependant, on observe des renversements de tendance récents: le Brésil et le Pérou sont devenus des pays d'émigration. L'inversion des processus se révèle particulièrement spectaculaire en Italie qui, après avoir laissé partir quelque 25 millions de ses enfants entre 1870 et 1970 , est devenue un des pays d'immigration les plus attractifs d'Europe.

Les départs massifs ont constitué des diasporas qui gardent des liens plus ou moins forts avec le pays d'origine. De 3 à 5 millions d'Iraniens vivent dans les États occidentaux. Quant au Salvador il possède 6240000 habitants et environ 3 millions de ressortissants installés à l'étranger, de sorte que l'émigration efface la croissance naturelle de la population. Quelque 45 millions de Chinois, soit l'équivalent de la population de l'Espagne, sont dispersés dans le monde. La diaspora comorienne, soit 370000 personnes, représente la moitié des habitants de I'Union des Comores ; 70000 de ces expatriés vivent clandestinement à Mayotte et constituent $40 \%$ de la population de ce département.

Les migrations internationales façonnent des nations ethniquement bigarrées et parfois déséquilibrées. Au Panama, le recensement de 2010 fait état de $55 \%$ de population métisse, $18 \%$ de noirs et mulâtres, $17 \%$ de blancs, $6 \%$ $d^{\prime}$ 'indigènes et $3 \%$ d'Asiatiques. L'Argentine est tellement construite par l'immigration transatlantique qu'elle est qualifiée de " terre d'Europe ". Le poids des immigrés apparaît particulièrement lourd dans les monarchies pétrolières : ils forment un tiers de la population saoudienne, $54 \%$ au Bahreïn, 90 \% dans les Émirats arabes unis et au Qatar.

Le livre souligne fortement les conséquences économiques et financières des phénomènes migratoires. Particulièrement importantes sont les remises, c'est-àdire les transferts financiers réalisés par les migrants vers leur pays d'origine. La
Chine et l'Inde qui reçoivent chaque année 60 à 70 milliards de dollars se disputent le premier rang mondial en la matière. Au Mexique, les remesas atteignent 23 milliards de dollars et dépassent les revenus du tourisme international. Dans certains pays, les envois de fonds, même $s^{\prime}$ ils s'avèrent moins importants, jouent un rôle essentiel : ils permettent auTadjikistan, pays le plus pauvre de l'ex-monde soviétique, de ne pas s'effondrer. Au Burkina Faso, les remises, 130 millions de dollars par an dont 102 en provenance de Côte d'Ivoire, constituent $10 \%$ du revenu national et font vivre de nombreuses familles.

La dimension psychologique et politique des migrations inspire des développements particulièrement intéressants. Certains pays refusent de se percevoir comme terres d'immigration ou d'émigration. La France et la Belgique ont longtemps compris l'arrivée des étrangers comme un phénomène conjoncturel. $\mathrm{Ce}$ dernier devient structurel en raison de sa persistance sur le long terme et de l'établissement définitif des immigrés, ce qui pose de délicats problèmes identitaires pour la société d'accueil comme pour les derniers venus. Les Égyptiens peinent à discerner les effets réels des migrations sur la transition démographique, le statut de la femme, les liens avec la communauté d'origine ; le cinéma et la littérature brossent le portrait de nombreux migrants égyptiens désorientés par l'expatriation, oubliant leurs valeurs et leur identité ou, après leur retour, introduisant dans le pays un islam wahhabite rigoriste qui constitue une menace larvée. La Russie qui fait état d'une diaspora de $\mathbf{3 0}$ millions de personnes, chiffre exagéré, essaie de garder un lien, voire un contrôle moral, sur ses compatriotes éloignés de leur patrie et, à cet effet, a créé des organisations vouées à cette action dans plus de cinquante pays. Cette politique rappelle les initiatives identiques prises dans l'entre-deux-guerres par les régimes autoritaires, celui de I'Italien Mussolini, du Polonais Pilsudski, de l'Espa- 
gnol Primo e Rivera. Ĺlnde, après avoir longtemps ignoré ses expatriés, se tourne vers eux, crée dans les années 2000 un ministère des Affaires des Indiens de l'étranger et octroie à des derniers un statut juridique, I'Overseas Citizenship of India, encourage ses ressortissants installés en Grande-Bretagne ou à Maurice à construire des bâtiments cultuels, vante les succès de ses hommes d'affaires et des écrivains de la diaspora, tout cela avec une volonté de reconnaissance géopolitique. En retour, les minorités établies à l'étranger, visibles ou non, peuvent susciter des réactions hostiles dans les pays d'accueil, surtout en période de difficultés économiques et de chômage. Ce fut le cas en France dans les années 1930 contre les Italiens, les Polonais, les Belges, les juifs et, depuis les années 1980, contre les Maghrébins. La xénophobie se déchaîne aussi contre les Zimbabwaéens d'Afrique du Sud tués lors des émeutes de 2008.

Les quelques exemples ici évoqués ne donnent qu'une image très imparfaite et incomplète de la richesse du dictionnaire. On ne peut émettre que deux réserves, non imputables aux auteurs : la bibliographie par pays est seulement consultable sur le site d'Armand Colin et la cartographie, résumée à dix cartes très générales, apparaît insuffisante, très certainement en raison du surcoût que l'éditeur aurait dû supporter. II n'en demeure pas moins que l'ouvrage rédigé par Gildas Simon et ses collègues constitue une réussite éclatante par sa qualité scientifique, sa clarté modèle, la mine d'informations et de réflexions qu'il offre. On peut désormais mesurer l'importance des migrations internationales à toutes les échelles, depuis le déplacement régional jusqu'au phénomène mondial, repérer les itinéraires séculaires, apprécier les comportements humains traditionnels ou conjoncturels, constater que les États conservent pour l'essentiel la maîtrise des politiques migratoires et des processus publics d'intégration. Les déplacements de population à l'échelle internationale dont les manifestations peuvent être, grâce à ce livre, définies dans le temps et l'espace, ce phénomène si important à notre époque devient très intelligible. Le système français d'enseignement qui associe l'histoire et la géographie vise à former ainsi des citoyens qui ne soient ni amnésiques ni aveugles. Le Dictionnaire des migrations internationales qui lie étroitement et brillamment les deux approches montre la pertinence de ce choix.

Ralph Schor

Professeur émérite d'histoire Université de Nice-Sophia-Antipolis 